\title{
Game Model of Thermal Power Capacity Planning Considering Environ- mental Constraint
}

\author{
Chen Weihua*, Liu Lianguang and Chen Yanwei
}

\author{
Key Laboratory of Alternate Electrical Power System with Renewable Energy Sources, North China Electric Power \\ University, Beijing 102206, China
}

\begin{abstract}
Rational planning of thermal power capacity is one of the main measures to reduce emissions. According to air pollution control plan, different environmental capacity regions provide different constraints to the scale of thermal power plants. With the development of electric market, unified relationship among thermal power plants has been changed to gambling one. To solve the above new problems emerged in the area of thermal power capacity planning, this paper presents a game model for thermal power capacity planning considering the constraint of environmental capacity. In this game-theory-based model, the thermal environmental adaptability factor, that reflecting the adaptability of environmental capacity to thermal power in different regions, is brought into the traditional profit function. Consequently, proper thermal power planning capacity adapting to environmental capacity could be obtained to provide support and basis for government regulation and enterprise decision. The effectiveness of the proposed model is validated by a series test results of different scale power planning examples.
\end{abstract}

Keywords: Game theory, environment adaptive, thermal power plant, planning, capacity.

\section{INTRODUCTION}

Recently, serious haze weather appeared in most areas of China is raising concerns about environmental protection and atmospheric pollution prevention, and how to plan thermal power capacity is becoming one of the related focus [1$3]$. Generally, optimization methods are adopted in the traditional thermal power capacity planning model. In [4-7], the total cost of investment, operation and maintenance, that relating to generation unit capacity, was chosen as objective function, and through searching its minimum value, the power capacity of the planning was obtained. However, the traditional planning model was unable to reflect the influence of thermal power development on environment. With further consideration of the requirements of environmental protection, [8] and [9] established a multi-objectives planning model with the minimum cost, waste gas and waste emissions. Although such model gives an optimal solution result that meets the conditions, it does not reflect the adaptability between environment capacity and the development of thermal power, as well as the interaction among thermal power plants.

In the market environment, every thermal power plant is an independent economic entity with rights and abilities of independent decision, and its basic goal is to maximize its own benefits. Driven by this endogenous power, a thermal power plant would select the most favorable action strategy based on its own actions and its competitors. The traditional thermal power planning model taking all power plants as a whole to optimize is no longer valid today, while game theory provides a method to solve the above problems $[10,11]$. The game model of thermal power planning can reflect influence and restriction among all the thermal power plants.

To solve the above problems, this paper starts with establishment of a game model of thermal power plants without environmental constraint, indicating an inevitable trend of excessive expansion of the scale of thermal power plant planning capacity. Then according to the Nash equilibrium solution of the model, environmental adaptability scale factor for thermal power plant is proposed to prevent excessive expansion. Finally a game model for thermal power plants considering constraint of environmental capacity is set up to ensure the adaptability between thermal power planning capacity and restriction of environment. Test results of different scale power planning examples show the effectiveness of this new proposed model.

\section{A GAME MODEL WITHOUT ENVIRONMENTAL CONSTRAINTS}

\subsection{The Prisoner's Dilemma of Capacity Planning}

For the convenience of analysis, hypothesis somewhere only two thermal power plants a and $\mathrm{B}$, and in all aspects of the two thermal power plants are quite. Thermal power plant thermal power plant is a and B power market competition of the two power plants, two coal-fired power plant has two strategies in the capacity planning: to further expand the capacity or not to expand capacity. Due to the environmental 
Table 1. User economic parameters.

\begin{tabular}{|c|c|c|c|}
\hline \multicolumn{2}{|c|}{} & \multicolumn{2}{|c|}{ Thermal Power Plant B } \\
\hline \multicolumn{2}{|c|}{} & Expansion & No Expansion \\
\hline \hline \multirow{2}{*}{$\begin{array}{c}\text { Thermal power } \\
\text { plant A }\end{array}$} & Expansion & $(\mathrm{b}, \mathrm{b})$ & $(\mathrm{c}, \mathrm{d})$ \\
\cline { 2 - 4 } & No expansion & $(\mathrm{d}, \mathrm{c})$ & $(\mathrm{a}, \mathrm{a})$ \\
\hline
\end{tabular}

capacity is certain, two coal-fired power plant if not further expand capacity, with each power plant gains for a; on the contrary, if two thermal power plant to further expand the capacity, then the deterioration of the environment and affect the two thermal power plant thermal power plant set each income, income is b; in addition, two coal-fired power plant if a thermal power plant to further expand the capacity of a coal-fired power plant, the other not to expand capacity, further expand the capacity of thermal power plant's profits will be more than not to expand the capacity of thermal power plant yield, set to further expand the capacity of thermal power plant of gains for the $\mathrm{C}$, not to expand the capacity of thermal power plant of gains for the $D$, see $d<b<a<c$, the game model as shown in the following Table (1).

For the convenience of analysis, hypothesis somewhere only two thermal power plants a and B, and two thermal power plant at all in this game, the two thermal power plant are fully rational, and information symmetry, each other fully understand each other's choice of strategy. In thermal power plant using a choice to further expand when capacity strategy, for thermal power plant for further expansion of B, if you choose the capacity gains of $\mathrm{B}$, if you choose not to expand the capacity gains of $D$, because the $d<b$ thermal power plant, so B will choose to further expand the capacity. In the thermal power plant a choice not to expand the capacity of the strategy used when, for thermal power plant for further expansion of B, if you choose the capacity gains of C, if you choose not to expand the capacity gains of a, because the $\mathrm{a}<\mathrm{c}$ thermal power plant, so B will choose to further expanding capacity. And vice versa, thus $(\mathrm{B}, \mathrm{b})$ is the Nash equilibrium of the game model of the solution, the two power plant will further expand the capacity, thus caught in a typical "prisoner's dilemma" [14,15].

\subsection{Benefit Analysis}

By the analysis of a section that, further expand the capacity of endogenous force in environmental constraint conditions of thermal power plant. Then the thermal power plant planning capacity and environmental constraints is whether to adapt to the problems that need further study. A thermal power plant planning capacity of a and B respectively and, due to the environmental carrying capacity is limited, the thermal power plant a and B unit of output power planning is decreasing function of the total capacity, thermal power plant unit capacity of a and $\mathrm{B}$ cost is constant, then the thermal power plants a and B revenue function respectively:

$$
\begin{aligned}
& U_{1}=P_{1} \times V\left(P_{1}+P_{2}\right)-C \times P_{1} \\
& U_{2}=P_{2} \times V\left(P_{1}+P_{2}\right)-C \times P_{2}
\end{aligned}
$$

Thermal power plant a and $\mathrm{B}$ revenue maximization strategy conditions for:

$$
\begin{aligned}
& \partial U_{1} / \partial P_{1}=0 \\
& \partial U_{2} / \partial P_{2}=0
\end{aligned}
$$

The specific form of a thermal power plant a and B the unit of output function is constant, the unit capacity cost, data with no units normalized value, EQ (1) to (4), while the thermal power plant a and B planning capacity response function respectively:

$$
\begin{aligned}
& P_{1}=47.5-0.5 P_{2} \\
& P_{2}=47.5-0.5 P_{1}
\end{aligned}
$$

By formula (5) and (6) to obtain power plant planning capacity of a and B are, the total capacity of thermal power plan. The type (1) and type (2) returns a and B in thermal power plant are, total revenue planning capacity for fire power plant.

If considered from the perspective of global optimization, a global optimal power planning a total capacity of, then the global power payoff function is:

$$
U_{0}=P_{0} \times V\left(P_{0}\right)-C \times P_{0}
$$

The type (7), and to get the global optimal power planning a total capacity, EQ (7) to get the global optimal power total revenue.

The process and results of the analysis can be extended to $\mathrm{n}$ thermal power plant capacity planning situation. Generally speaking, in the market environment, when a $\mathrm{n}$ coal-fired power plant, the game is formed between the n power plant capacity planning. If there is no environmental constraints, each power plant will choose to expand the capacity of the strategy, the final environmental resources will be depleted. However, compared with the method of global optimal, each power plant will eventually get lower income. But as the thermal power plants are trapped in a "prisoner's dilemma", oneself is unable to solve this problem, we must introduce factor of the environmental adaptability to support external supervision measures to solve the problem.

\section{THE GAME MODEL OF ENVIRONMENTAL CON- STRAINTS INTO ACCOUNT}

\subsection{The Adaptive Function}

The root cause of these problems is the thermal power plant capacity planning does not consider the environmental cost, which does not reflect the thermal power plant capacity planning for the restriction of environment adaptability, and relevant departments to supervise. A method to solve this problem is the measurement of capacity planning of environmental adaptability, and to provide evidence for the supervision department.

This paper presents the adaptability to environment factor to solve the above problem. The following design principle of environmental adaptability factor items: 
The adaptability to environment factor to reflect regional differences in the environment. At present, China's environmental protection situation is grim, with regional atmospheric environmental problems the respirable particulate matter pollutants increasingly prominent characteristics. Overall, environmental quality in the west to the eastern area is better than that of Beijing Tianjin Hebei, Yangtze River Delta, Pearl River Delta region, such as the great environment pressure. Environmental adaptability factor should reflect these differences, resulting in the areas under environmental stress strictly constrained thermal power capacity planning, and be able to use the good environment quality area of storage space to solve the power supply problem.

3) the adaptability to environment factor to consider in thermal power plant acceptance, ensure stability of the policy execution. Environmental protection is a systems engineering project, the extensive participation of all walks of life and the need to vigorously with the. Occupation of the environmental capacity of the thermal power plant emissions is a part of environmental protection engineering, therefore in the first satisfy the environment constraints, also want to consider from the angle of sustainable development of thermal power plant, the constraint intensity should be appropriate.

\subsection{The Establishment of the Model}

Located in the market environment, a certain area of $n$ power plants. The planning capacity of thermal power plant I respectively, the total capacity of power planning area. As the thermal power emissions to the environment destruction, unit capacity output in the area of power plant is a decreasing function of the total capacity of power planning. Capacity of thermal power units in the region is a constant factor cost, environmental adaptability, profit function for thermal power plant i:

$U_{i}=P_{i} \times V\left(P_{1}+\cdots P_{i}+\cdots+P_{n}\right)-C \times P_{i}-\operatorname{Adp}\left(P_{i}\right)$

Thermal power plant I revenue maximization strategy conditions for:

$\partial U_{i} / \partial P_{i}=V+P_{i} \times V^{\prime}-C-A d p^{\prime}=0$

By formula (9) shows: for thermal power plant I, if we do not consider the environmental constraints, then each additional unit of capacity planning, will produce positive, anti role in two aspects: on the one hand because of the capacity increase of the expansion income $\mathrm{V}$; on the other hand, due to the deterioration of the environment to reduce the income. Compared with and without consideration of environmental constraints, if considering environmental constraints, then it should also consider the environmental cost, to adapt to the environment here is expressed as a function of. According to the design principle of the fitness function, adaptive function can be designed as a piecewise function or exponential function forms. The adaptive function of piecewise function can be expressed as:

$$
A d p=\left\{\begin{array}{cc}
A_{1} \times P_{i}^{2} & \text { env_low } \\
A_{2} \times P_{i}^{2}, & \text { env_mid } \\
A_{3} \times P_{i}^{2}, & \text { env_high }
\end{array}\right.
$$

Type: said the environmental capacity is relatively small, said the environmental pressure capacity of medium, said the environmental pressure capacity is bigger. As the coefficient corresponding to different environment capacity, including.

The adaptive function of exponential function can be expressed as:

$$
A d p=A \times \exp \left(P_{i}\right)
$$

\subsection{The Algorithm Flow}

Process of this algorithm is as below. Step one: input system and economic parameters, and according to the characteristics of regional environmental capacity selection of environmental adaptability factor; step two: the establishment of the thermal power plant capacity planning game model of environmental constraints into account, the formation of strategy combination; step three: the benefits of each solution strategy, reaction function; step four: the Nash equilibrium point to determine response function all thermal power plants based on game results, output, capacity planning scheme of thermal power plant.

\section{CASE STUDY}

Set up a regional development in power planning, this paper will respectively with consideration of environmental constraints game model of thermal power capacity planning and environmental constraints game model algorithm considering the calculation, through comparison of the results showed the feasibility of the proposed method contrast.

\subsection{Do not Take into Account Environmental Con- straints}

Unit of output set fire power plant thermal power planning is a decreasing function of the total capacity, the specific form, $\mathrm{n}$ is the number of thermal power plant in the game. Constant power plant unit capacity cost is C. Data with no units normalized value, when, $n=2$, reaction function curve of two thermal power plant as shown in Fig. (1).

Solid line in the figure is a reflection of a function curve of thermal power plant, the dotted line is a reflection of function curve of thermal power plant B, the Nash equilibrium of their point of intersection is the two thermal power plant capacity planning solution. Figure triangular marker is two thermal power plant capacity planning for the global optimal solution, the planning result is visible Nash equilibrium results than the global optimal planning.

\section{1) Influence the number of thermal power plant}

Thermal power plant is the player in the game, when the number of thermal power plants in the game by 2 to 20 , the total income of each thermal power plant planning capacity change and power planning capacity as shown in Fig. (2).

Planning capacity corresponding circle mark points in the view in the game of power plant different quantity of each fire power plant, the total revenue corresponding square mark points in the game when different number of thermal power plant thermal power planning capacity. With the increase of the number of visible power plant participation, 


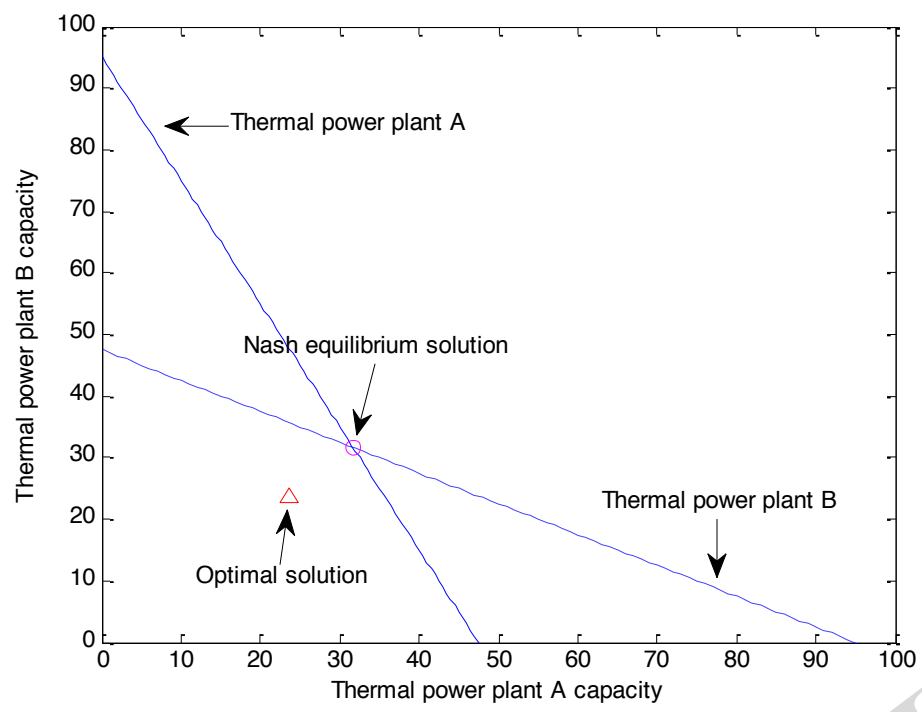

Fig. (1). Unconstrained Nash equilibrium solution.

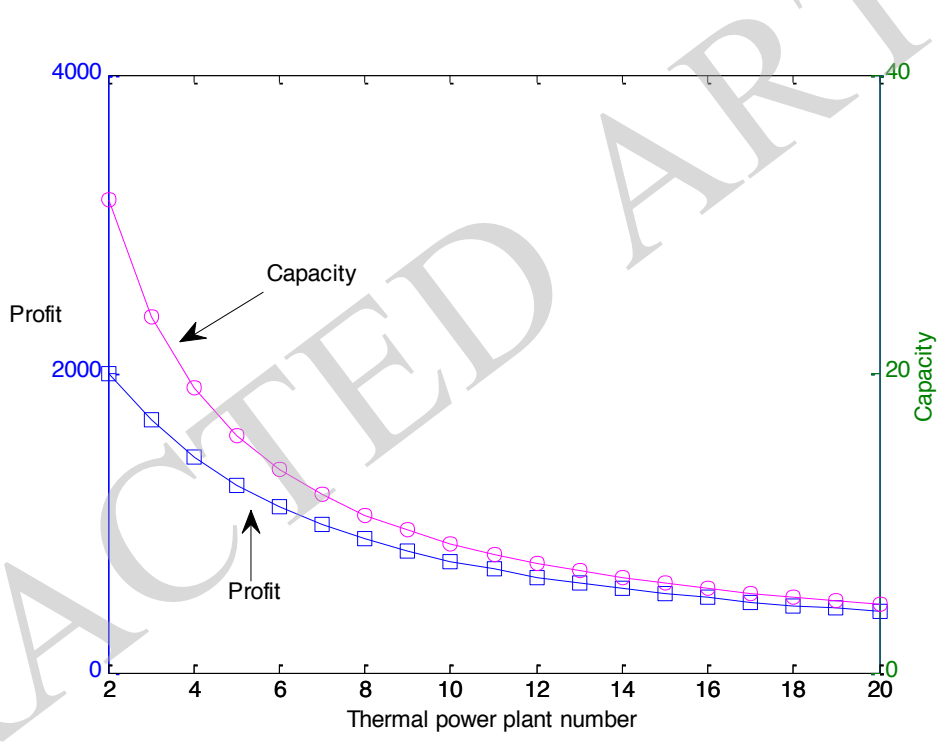

Fig. (2). Effects of plant number.

environmental resources will be over the low efficiency of the use of, and participation in thermal power plants are unable to get the real benefit.

\section{2) Effect of thermal power unit capacity cost}

Fixed the other parameters unchanged, when involved in the game of power plant unit capacity cost increased from 1 to 20 , the total income of each thermal power plant planning capacity change and power planning capacity as shown in Fig. (3).

The planning capacity of each fire power plant cost of thermal power plant unit capacity corresponding to different circle mark points in the view in the game when the power planning capacity, the total return cost of thermal power plant unit capacity corresponding to different square mark points in the game when the. Visible with the increase in thermal power plant unit capacity cost, total revenue power reduction, all thermal power plants tended to reduce capacity planning of their own.

\section{3) Effects of environmental capacity}

Fixed the other parameters, when the region's environmental capacity increased from 50 to 150 , the total income of each thermal power plant planning capacity change and power planning capacity as shown in Fig. (4).

The planning capacity of each fire power plant environmental capacity of circle marker points in the graph corresponding areas are not at the same time, the total revenue environment capacity square mark points corresponding regional power planning and capacity. Visible along with the increase of the regional environmental capacity, the total income of each thermal power plant capacity and power have increased, reflect only the natural and social harmonious development, in order to have a win-win basis. 


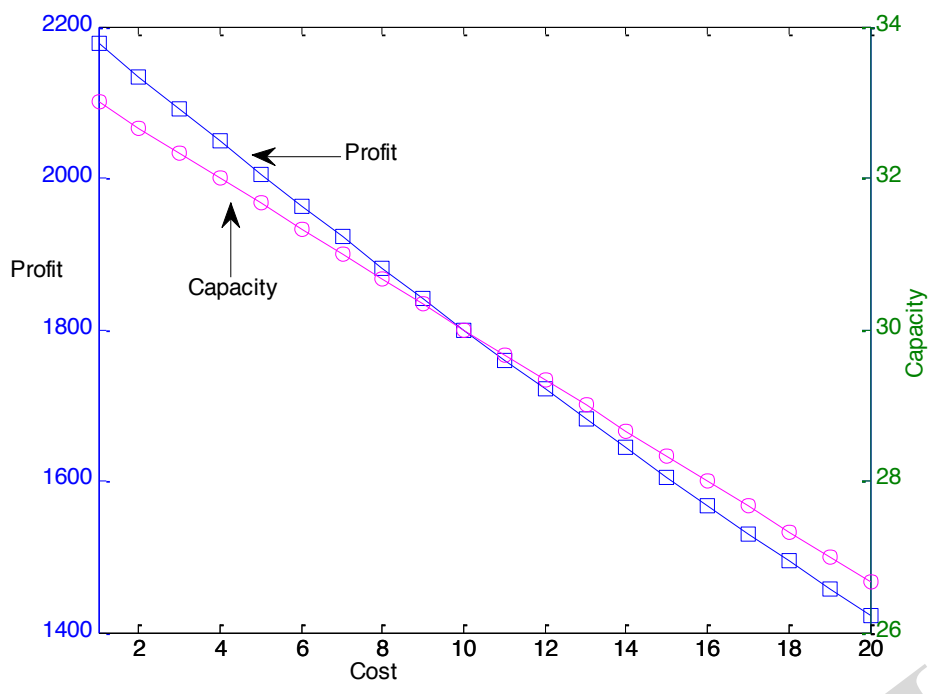

Fig. (3). Effect of power unit capacity cost.

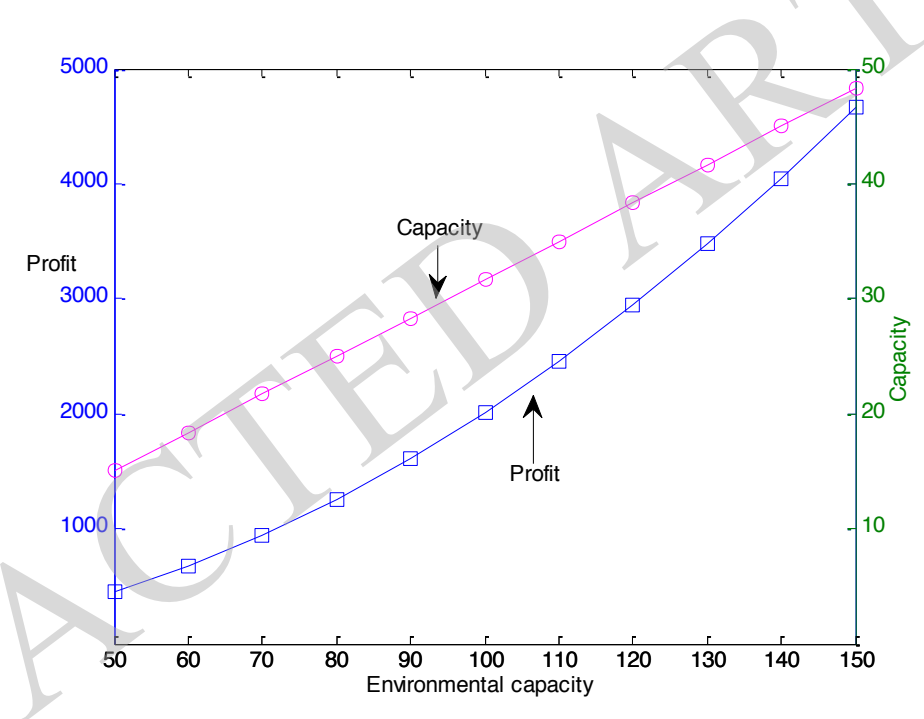

Fig. (4). Effect of environmental capacity.

\subsection{Consider the Environmental Constraints}

1) The same regional environmental capacity

Consider $\mathrm{n}=3$, where the game of power plant in regional environment capacity is the same, when the factor of the environmental adaptability of $\mathrm{A}=0.8$, the response function of three coal-fired power plants as shown in Fig. (5).

When $\mathrm{n}=3$, the reaction function by the curve into a surface; when the reaction function, will become a hypersurface, has been unable to display in the 3 dimension coordinate axis. The corresponding dark ball marker points in the graph is not a Nash equilibrium solution of the adaptability of environment factor, the corresponding light colored balls marked point has a Nash equilibrium solution of the adaptability of environment factor. Seen from the diagram, using environmental adaptability factor after effectively limits the excessive expansion of thermal power plant planning capacity.
Have certain effects on selected environmental adaptability factor values for the coal-fired power plant planning capacity, as shown in the following Table 2. As shown in the table, when $\mathrm{A}=0.8$, the Nash equilibrium solution is greater than the global optimal solution; when $\mathrm{A}=1.2$, the Nash equilibrium solution is less than the global optimal solution. At present, in choosing the value of A, but also more dependent on the management and operation department experience, further research is needed to improve the management of the selection method, accuracy, so as to Protect environment, reasonable needs and can not restrain power plant.

\section{2) Regional environmental capacity of different}

When the thermal power plant is more, a broader regional distribution, different regional environment capacity is different, so for the A to be divided into regional selection. Let $n=10,3$ regional 10 coal-fired power plants are distributed in a certain area, three regional environmental capacity of 


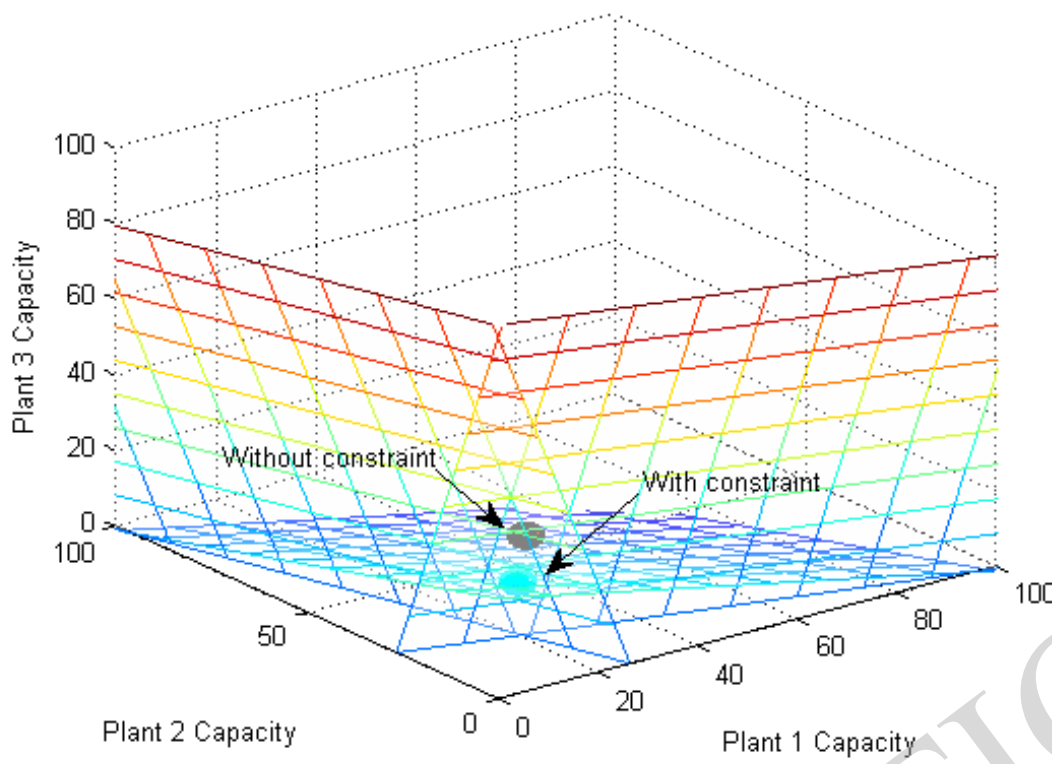

Fig. (5). Algorithm flow chart.

Table 2. System operation parameters.

\begin{tabular}{|c|c|c|c|}
\hline & \multirow{2}{*}{$\begin{array}{c}\text { The Optimal } \\
\text { Solution }\end{array}$} & \multicolumn{2}{|c|}{ Nash Equilibrium Solution } \\
\cline { 3 - 4 } & 15.8 & 16.9 & $\mathbf{A}=\mathbf{0 . 8}$ \\
\hline \hline $\begin{array}{c}\text { Thermal power } \\
\text { plant 1 }\end{array}$ & 15.8 & 16.9 & 14.8 \\
\hline $\begin{array}{c}\text { Thermal power } \\
\text { plant 2 }\end{array}$ & 15.8 & 16.9 & 14.8 \\
\hline $\begin{array}{c}\text { Thermal power } \\
\text { plant 3 }\end{array}$ & & & 14.8 \\
\hline
\end{tabular}

different, were low, medium or high, specific factor environmental adaptability of item values are: low environmental capacity, environmental capacity in the region, the regional environmental capacity and high area, planning capacity of each fire power plant are as follows Table $\mathbf{3}$ lists shown.

As shown in the table above, through the different areas of environmental capacity, thermal power capacity planning appeared difference results. Thermal power capacity planning of regional environment capacity low reduction, thermal power capacity planning of regional environment capacity high increase. The model reflects the "action plan" for prevention and control of atmospheric pollution in the "basic principles and ideas of carrying out the difference of central and western regions, the eastern part of the energy policy".

\section{CONCLUSION}

1) Factor power environmental adaptability is proposed in this paper, reflects the different requirements for thermal adaptability to different environmental capacity region, the
Table 3. System operation parameters.

\begin{tabular}{|c|c|c|c|}
\hline \multirow[b]{2}{*}{$y$} & \multirow{2}{*}{$\begin{array}{c}\text { Environmental } \\
\text { Capacity }\end{array}$} & \multicolumn{2}{|c|}{ Nash Equilibrium Solution } \\
\hline & & $\begin{array}{c}\text { Different } \\
\text { Environmental } \\
\text { Capacity }\end{array}$ & $\begin{array}{c}\text { Same } \\
\text { Environmental } \\
\text { Capacity }\end{array}$ \\
\hline $\begin{array}{c}\text { Thermal power } \\
\text { plant } 1\end{array}$ & Low & 11.7 & 8.7 \\
\hline $\begin{array}{c}\text { Thermal power } \\
\text { plant } 2\end{array}$ & Low & 11.7 & 8.7 \\
\hline $\begin{array}{c}\text { Thermal power } \\
\text { plant } 3\end{array}$ & Low & 11.7 & 8.7 \\
\hline $\begin{array}{c}\text { Thermal power } \\
\text { plant } 4\end{array}$ & Media & 8.7 & 8.7 \\
\hline $\begin{array}{c}\text { Thermal power } \\
\text { plant } 5\end{array}$ & Media & 8.7 & 8.7 \\
\hline $\begin{array}{c}\text { Thermal power } \\
\text { plant } 6\end{array}$ & Media & 8.7 & 8.7 \\
\hline $\begin{array}{c}\text { Thermal power } \\
\text { plant } 7\end{array}$ & Media & 8.7 & 8.7 \\
\hline $\begin{array}{c}\text { Thermal power } \\
\text { plant } 8\end{array}$ & High & 5.8 & 8.7 \\
\hline $\begin{array}{c}\text { Thermal power } \\
\text { plant } 9\end{array}$ & High & 5.8 & 8.7 \\
\hline $\begin{array}{c}\text { Thermal power } \\
\text { plant } 10\end{array}$ & High & 5.8 & 8.7 \\
\hline
\end{tabular}


distinction of thermal power development strategy in different regions, the coordinated development of power construction and environmental protection.

2) Considering thermal power capacity planning game model of environmental capacity constraints is established in this paper, the application of response function communication game relation between each power plant, which reflects the rights and ability of market environment of power plant independent decision-making, in order to provide support and basis for the supervision of government and business decision, ensure the smooth implementation of the planning results.

3) Example analysis shows that the effectiveness of the proposed model. In addition, in order to improve the accuracy of calculation on the environmental capacity constraints, will be selected to power the adaptability to environment factor in further study.

\section{CONFLICT OF INTEREST}

The authors confirm that this article content has no conflicts of interest.

\section{ACKNOWLEDGEMENTS}

Declared none.

\section{REFERENCES}

[1] D. Singh, and K. S. Verma, "Multi-objective Optimization for DG planning with models," IEEE Trans on Power Systems, vol. 24, pp. 427-436, 2009,
[2] P. Zhang, W. Li, and S. Li, "Reliability assessment of photovoltaic power systems: Review of current status and future perspectives," Applied Energy, vol. 104, pp. 822-833, 2013.

[3] C. H. Lin, W. L. Hsieh, and C. S. Chen, "Optimization of photovoltaic penetration in distribution systems considering annual duration curve of solar irradiation," IEEE Transactions on Power Systems, vol. 27, pp. 1090-1097, 2012.

[4] J. Wang, B. Bao, and J. Xu, "Dynamical effects of equivalent series resistance of output capacitor in constant on-time controlled buck converter," IEEE Transactions on Industrial Electronics, vol. 60, pp. 1759-1768, 2013.

[5] W. C. Chen, C. S. Wang, and Y. P. Su, "Reduction of equivalent series inductor effect in delay-ripple reshaped constant on-time control for buck converter with multilayer ceramic capacitors," IEEE Transactions on Power Electronics, vol. 28, pp. 2366-2376, 2013.

[6] J.A. Martinez, and J. Martin-Arnedo, "Voltage sag stochastic prediction using an electromagnetic transients program," IEEE Transactions on Power Systems, vol. 19, pp. 1005-1014, 2004.

[7] J.D. McCalley, V. Vittal, and N. Abi-Samra, "An overview of risk based security assessment," IEEE Power Engineering Society Summer Meeting, vol. 1, pp. 173-178, 1999.

[8] T. Qian, W. K. Wu, and W. D. Zhu, "Effect of combined output capacitors for stability of buck converters with constant on-time control," IEEE Transactions on Industrial Electronics, vol. 60, pp. 5585-5592, 2013.

[9] T.. Konjic, V. Miranda, and I. Kapetanovic, "Fuzzy inference systems applied to LV substation load estimation," IEEE Transactions on Power Systems, vol. 20, pp. 742-749, 2005.

[10] H.. Wan, J.D. McCalley, and V. Vittal, "Increasing thermal rating by risk analysis," IEEE Transactions on Power Systems, vol. 14, pp. 815-828, 1999.

[11] Y. Yan, F. C. Lee, and P. Mattavelli, "Comparison of small signal characteristics in current mode control schemes forpoint-of-load buck converter applications," IEEETransactions on Power Electronics, vol. 28, pp. 3405-3414, 2013.

(C) Weihua et al.; Licensee Bentham Open.

This is an open access article licensed under the terms of the Creative Commons Attribution Non-Commercial License (http://creativecommons.org/licenses/bync/3.0/) which permits unrestricted, non-commercial use, distribution and reproduction in any medium, provided the work is properly cited. 